\title{
Nephrobronchial fistula. A case report and review of the literature
}

\section{Jonathan A. Harikrishnan, Thomas C. Hall, Simon J. Hawkyard}

Department of Urology, Scarborough General Hospital, Scarborough, North Yorkshire, United Kingdom

\section{KEY WORDS}

nephrobronchial fistula pyonephrosis D

nephrectomy xanthogranulomatous pyelonephritis

\section{ABSTRACT}

Nephrobronchial fistulas are a rare complication of xanthogranulomatous pyelonephritis. We report the diagnosis of such a case together with its investigation, management and a review of current literature.

\section{CASE REPORT}

A 37-year-old lady presented to our department with an incidental large left sided renal calculus discovered on routine antenatal ultrasonography. This was asymptomatic and non-obstructing and therefore treated conservatively. She had a background of recurrent urinary tract infection but no other co morbidities. Shortly after the birth of her second child she was admitted acutely to our department with left loin pain and pyrexia. An ultrasound was performed and an obstructing upper tract calculus identified. This required percutaneous nephrolithotomy. She subsequently had a parathyroidectomy for a benign adenoma causing hypercalcemia.

The patient, aged 45, was admitted acutely with left sided loin pain, pyrexia, and purulent urine. Renal ultrasound, cystoscopy, ureteroscopy, and retrograde ureterogram were performed. They demonstrated a large multiloculated collection around a non-functioning left kidney. A plan

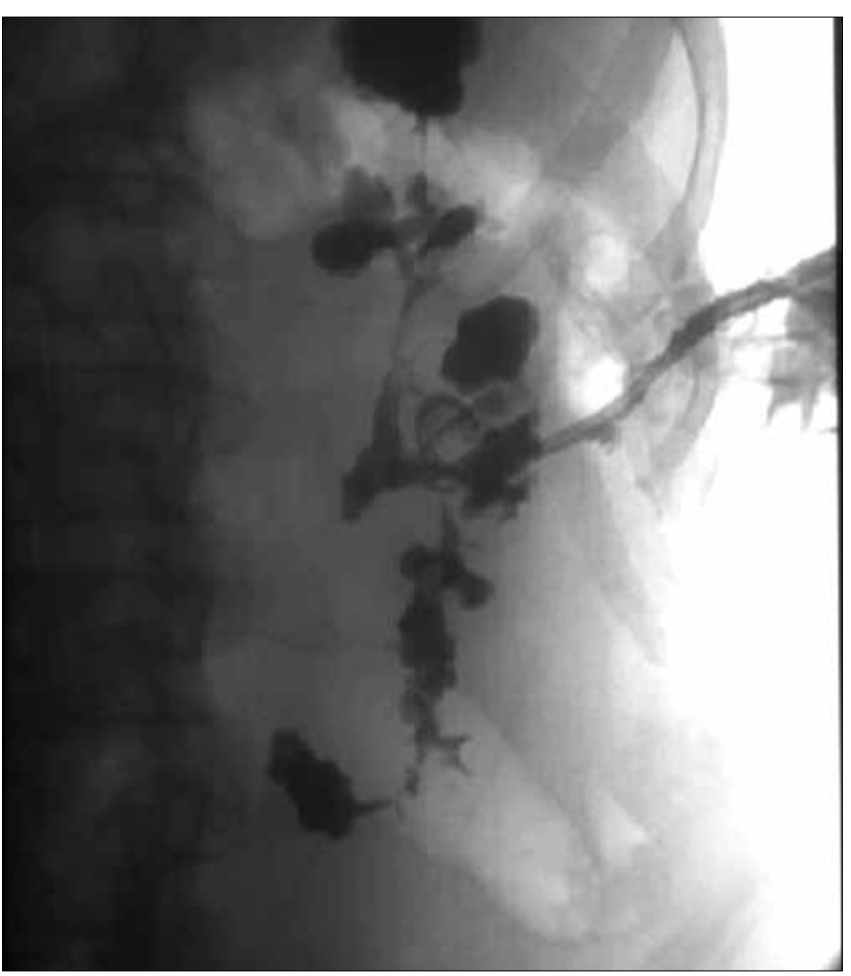

Fig. 1. Nephrostogram. was made to perform a left nephrectomy. This was not completed, however, as dissection proved difficult secondary to intraoperative hemorrhage. Instead open abscess drainage was performed. Postoperative recovery was unremarkable and she was discharged home five days later.

After six years she was admitted on three occasions with recurrent pyrexia, rigors, and suspected urinary tract infections over the course of a month. None of these required intervention other than with antibiotics. Blood tests were unremarkable, urine cultures were sterile, and ultrasound reported as normal. An outpatient abdominopelvic CT was organized and revealed a swollen left kidney with ureteric obstruction. A left nephrostomy was inserted. Proteus species was cultured in the aspired pus. She improved with antibiotic therapy.

A nephrostogram was performed and at that time the patient complained of a bitter taste in her mouth and expectorated contrast fluid. The nephrostogram revealed contrast crossing the diaphragm in accordance with a nephrobronchial fistula (Figs. 1, 2, 3).

She underwent a left nephrectomy and the fistula tract was closed, histology confirmed this was xanthogranulomatous pyelonephritis. She underwent an uncomplicated post-operative course and was discharged home.

\section{DISCUSSION}

Xanthogranulomatous pyelonephritis (XGP) is an atypical chronic inflammatory disorder of the kidney. It is characterized by a destructive mass that leads to an infectious renal phlegmon. Parsons et al. described XGP as an invasive disease that commonly fistulates with adjacent structures [1]. It is frequently associated with repeated ureteric obstruction, infection, and immunocompromise. Our patient grew a culture positive

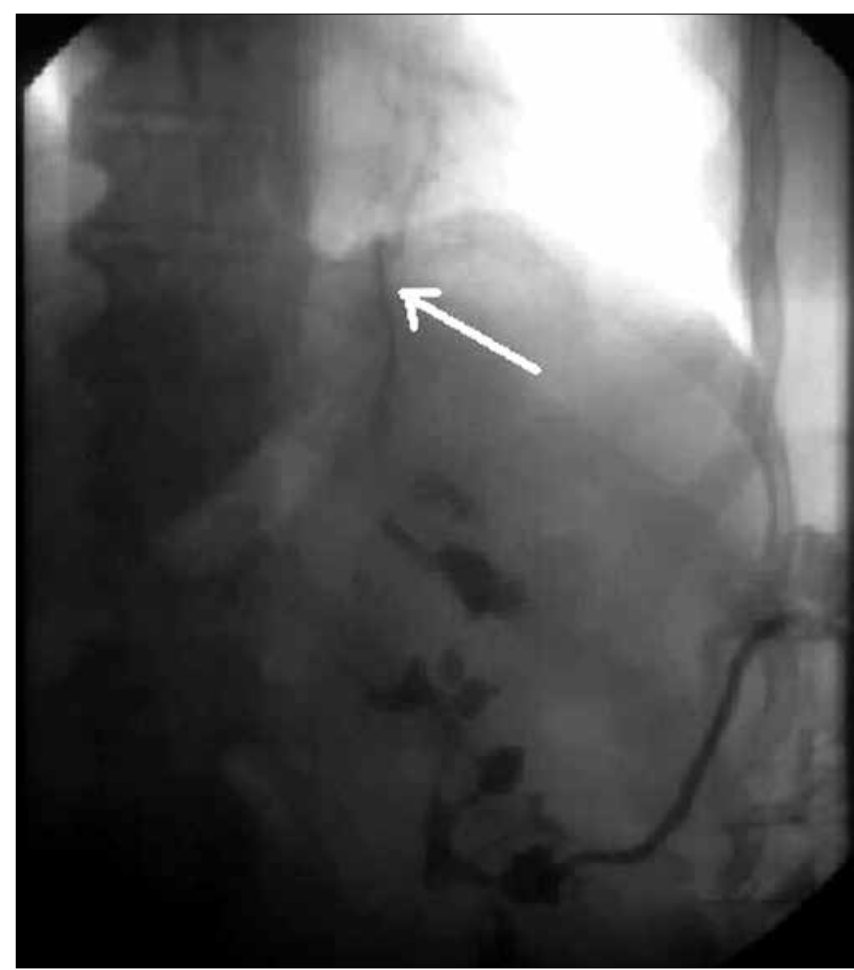

Fig. 2. Nephrostogram (fistulous tract indicated by white arrow). 


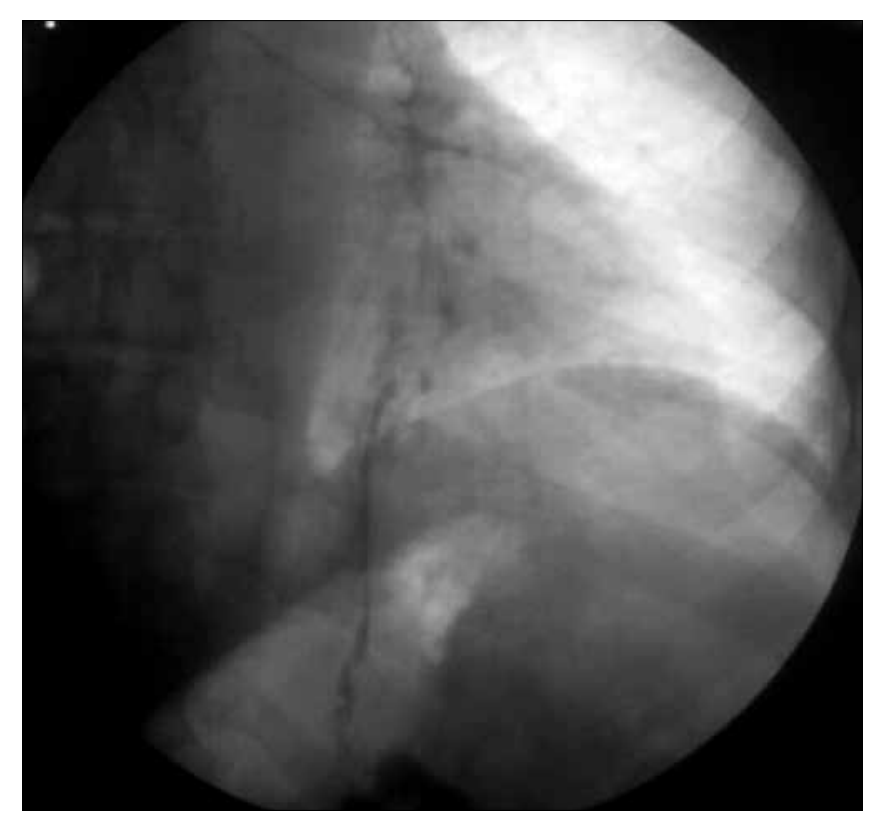

Fig. 3. Nephrostogram demonstrating thoracic contrast medium.

for Proteus and, indeed, this organism together with the Escherichia coli species account for a significant number of cases [2].

A Medline search revealed 31 reported cases worldwide of nephrobronchial fistula since 1900 . Frequently it is associated with nephrolithiasis and XPG. In addition to thoracic spread, fistulas have been reported cutaneously to the duodenum and colon. Evans et al. described that the direction of superior retroperitoneal renal spread of XPG is by lines of renal fascial fusion planes [3]. Alifano et al. described the lumbosacral trigone as a relatively weak area of the diaphragm that may transmit infection [4].

Management of XPG and subsequent nephrobronchial fistula can be conservative with antibiotic therapy, but ultimately requires surgical drainage and nephrectomy ${ }^{5}$.

Our case report demonstrates the difficulties encountered in diagnosing this rare condition. It should be suspected in any patient with a pyonephrosis from obstructing nephrolithiasis in combination with thoracic symptoms.

\section{REFERENCES}

1. Parsons MA, Harris SC, Grainger RG et al: Fistula and sinus formation in xanthogranulomatous pyelonephritis. A clinico-pathological review and report of four cases. BJU 1986; 58: 488-493.

2. Korkes F, Fawretto RL, Bròghio $\mathrm{M}$ et al: Xanthogranulomatous pyelonephritis. Clinical experience with 41 cases. Urology 2008; 71 (2): 178-180.

3. Evans JA, Meyers MA, Bosniak MA: Acute renal and perirenal infections. Semin Roentgenol 1971; 6: 276-291.

4. Alifano M, Venissac N, Chevallier D et al: Nephrobronchial fistula secondary to xanthogranulomatous pyelonephritis. Ann Thorac Surg 1999; 68: 1836-1837.

5. O'Brien JD, Ettinger NA: Nephrobronchial fistula and lung abscess resulting from nephrolithiasis. Chest 1995; 108: 1166-1168.

\section{Correspondence}

Jonathan A. Harikrishnan

Department of Urology, Scarborough General Hospital

Woodlands Drive, Scarborough

North Yorkshire Y012 60L

phone: +447850136211

j.a.harikrishnan@hotmail.co.uk 